\title{
N-Acetylglucosamine (GlcNAc) Sensing, Utilization, and Functions in Candida albicans
}

\author{
Han Du ${ }^{1,2}\left(\right.$, Craig L. Ennis ${ }^{3,4}{ }^{-}$, Aaron D. Hernday ${ }^{3}$, Clarissa J. Nobile ${ }^{3 \oplus}$ and \\ Guanghua Huang 1,*(D) \\ 1 State Key Laboratory of Genetic Engineering, School of Life Sciences, Fudan University, \\ Shanghai 200438, China; handu@fudan.edu.cn \\ 2 Institutes of Biomedical Sciences, Fudan University, Shanghai 200438, China \\ 3 Department of Molecular and Cell Biology, University of California, Merced, Merced, CA 95343, USA; \\ cennis@ucmerced.edu (C.L.E.); ahernday@ucmerced.edu (A.D.H.); cnobile@ucmerced.edu (C.J.N.) \\ 4 Quantitative and Systems Biology Graduate Program, University of California, Merced, \\ Merced, CA 95343, USA \\ * Correspondence: huanggh@fudan.edu.cn; Tel./Fax: +86-21-3124-6792
}

Received: 8 July 2020; Accepted: 29 July 2020; Published: 7 August 2020

\begin{abstract}
The sensing and efficient utilization of environmental nutrients are critical for the survival of microorganisms in environments where nutrients are limited, such as within mammalian hosts. Candida albicans is a common member of the human microbiota as well as an opportunistic fungal pathogen. The amide derivative sugar $\mathrm{N}$-acetlyglucosamine (GlcNAc) is an important signaling molecule for $\mathrm{C}$. albicans that could be a major nutrient source for this fungus in host settings. In this article, we review progress made over the past two decades on GlcNAc utilization, sensing, and functions in C. albicans and its related fungal species. GlcNAc sensing and catabolic pathways have been intensively studied in C. albicans. The C. albicans protein Ngt1 represents the first identified GlcNAc-specific transporter in eukaryotic organisms. In C. albicans, GlcNAc not only induces morphological transitions including the yeast to hyphal transition and the white to opaque phenotypic switch, but it also promotes fungal cell death. The Ras-cAMP/PKA signaling pathway plays critical roles in regulating these processes. Given the importance of GlcNAc sensing and utilization in C. albicans, targeting GlcNAc associated pathways and key pathway components could be promising in the development of new antifungal strategies.
\end{abstract}

Keywords: cell surface receptor; Ngt1; N-acetylglucosamine; GlcNAc; GlcNAc utilization; morphological transitions; virulence; cAMP signaling; Candida albicans

\section{Introduction}

The amide derivative sugar $\mathrm{N}$-acetlyglucosamine (GlcNAc) is a component of the peptidoglycan layer of bacterial cell walls and the chitin layer of fungal and parasitic cell walls [1]. It is also found in the extracellular matrix of bacterial biofilms and in the extracellular matrix glycosaminoglycans of animal cells [1]. GlcNAc is a nutrient source for many microorganisms and also plays critical roles in signaling for both bacterial and mammalian cells [1-3]. It regulates a variety of biological processes including morphological transitions, virulence, response to oxidative stresses and antifungal agents, and cell death in several fungal species such as Candida albicans [1,4-7], Candida tropicalis [8-10], Yarrowia lipolytica [11], and Histoplasma capsulatum [12]. Candida species are common members of the human microbiota; however, they are also opportunistic pathogens that can cause superficial and life-threatening infections in humans, especially in immunocompromised individuals [13-16]. Given the ubiquitous nature of GlcNAc in mammalian and microbial cells, it seems likely that GlcNAc 
would be abundant in the human host and could serve as a critical signaling molecule that regulates the switch between commensalism and pathogenicity for the Candida species. Here, we review the current knowledge of GlcNAc sensing, utilization, and functions in the pathogenic Candida species.

\section{Ngt1, the GlcNAc-Specific Transporter}

A comparative proteomic analysis of $C$. albicans plasma membrane localized proteins from yeast and filamentous cells induced by GlcNAc identified the protein Ngt1 (N-acetylglucosamine-specific transporter 1) as a GlcNAc-specific transporter [17], representing the first eukaryotic GlcNAc-specific transporter to be identified. Ngt1 is comprised of 12 transmembrane domains and has structural similarity to the major facilitator superfamily (MFS) membrane transporters. Although both serum and GlcNAc are potent inducers of hyphal development, only GlcNAc was found to induce Ngt1 in C. albicans hyphal cells [17]. Interestingly, Ngt1 was also found to be induced by the engulfment of C. albicans cells by macrophages [17]. Consistent with the latter finding, transcriptomic analysis showed that the expression of NGT1 and GlcNAc catabolism-associated genes were upregulated upon macrophage engulfment [18]. Taken together, these observations suggest that GlcNAc sensing and signaling are likely intertwined with the ability of $C$. albicans to interact with and evade the host immune response.

Saccharomyces cerevisiae, a distantly related yeast species, does not contain an ortholog of NGT1 and is unable to utilize GlcNAc as a sole carbon source. Heterologous expression of C. albicans NGT1 in S. cerevisiae, however, can promote GlcNAc uptake, further confirming the role of Ngt1 as a GlcNAc transporter $[17,19]$. Interestingly, a BLASTP search of C. albicans Ngt1 identifies homologs in diverse organisms across fungi, plants, and metazoans, highlighting the importance of GlcNAc sensing and utilization in eukaryotes $[12,17,20,21]$. For example, the dimorphic Fungus Histoplasma capsulatum has two GlcNAc transporters that are homologous to C. albicans Ngt1 and are encoded by NGT1 and NGT2 [12]. H. capsulatum Ngt1 and Ngt2 are required for efficient filamentous growth induced by GlcNAc, suggesting that mechanisms of GlcNAc sensing and transport are conserved in fungi. Interestingly, in the maize plant, Zea mays, Nope1 was identified as the first plasma membrane GlcNAc transporter in plants [20]. Ectopic expression of the NOPE1 gene from Z. mays in a C. albicans ngt1/ngt1 mutant strain was able to restore cell and filamentous growth of the C. albicans ngt1/ngt1 mutant strain on GlcNAc-containing medium, suggesting that the functions of GlcNAc transporters are conserved across plant and fungal kingdoms [20].

\section{GlcNAc Catabolism}

GlcNAc is catabolized to fructose-6-phosphate in a stepwise manner by GlcNAc kinase (EC 2.7.1.59), GlcNAc-6-phosphate deacetylase (EC 3.5.1.33), and glucosamine-6-phosphate deaminase (EC 3.5.99.6), which are encoded by HXK1, DAC1, and NAG1, respectively (Figure 1) $[19,22,23]$. The presence of GlcNAc rapidly induces the expression of all three genes that are clustered together in the C. albicans genome $[19,22]$. This clustering of associated genes with related functions is common in microorganisms and may facilitate their coordinated regulation in response to environmental changes.

Recently NGS1, which encodes an N-acetyltransferase related to the histone acetyltransferase Gcn5, was identified as a GlcNAc sensor in C. albicans [24,25]. Ngs1 is constitutively targeted to the promoters of GlcNAc-inducible genes via the transcription factor Rep1 and is required for their transcriptional activation in response to GlcNAc. Deletion of either NGS1 or REP1 in C. albicans completely abolishes fungal growth on medium containing GlcNAc as the sole carbon source. GlcNAc also induces the expression of $H E X 1$, which encodes a secreted $\mathrm{N}$-acetylglucosaminidase that is required for virulence and for C. albicans GlcNAc scavenging during an infection [26,27]. The $\mathrm{N}$-acetylglucosaminidase activity is abolished by mutation of NGS1 in C. albicans cells in the presence of GlcNAc, suggesting that the expression of HEX1 is controlled in an Ngs1-dependent transcriptional induction manner [24,25] (Figure 1). Another transcription factor, Ron1, was discovered to regulate GlcNAc catabolism and GlcNAc-induced filamentous growth in C. albicans [24]. This result is in contrast to the findings in a 
recent study, where the deletion of RON1 using the CRISPR/Cas9 method did not affect filamentous growth in response to GlcNAc [28]. However, other phenotypes were indeed observed for the ron1/ron1 mutant strain in this study that were consistent with its role in GlcNAc catabolism [28]. Moreover, the $n d t 80 / n d t 80$ ron1/ron1 double mutant strain exhibited increased filamentation in the presence of GlcNAc. Taken together, these results suggest that Ron1 could function as a negative regulator of filamentation in the absence of Ndt80 [28]. The discrepancies between the two studies could be due to secondary mutations that may have arisen during construction of the different ron $1 /$ ron 1 mutant strains used. In Trichoderma reesei, a non-pathogenic environmental fungal species distantly related to the Candida species, a Ron1 ortholog also controls the expression of a GlcNAc catabolic gene cluster [21], suggesting that the regulation of GlcNAc responsive genes is conserved across the fungal kingdom.

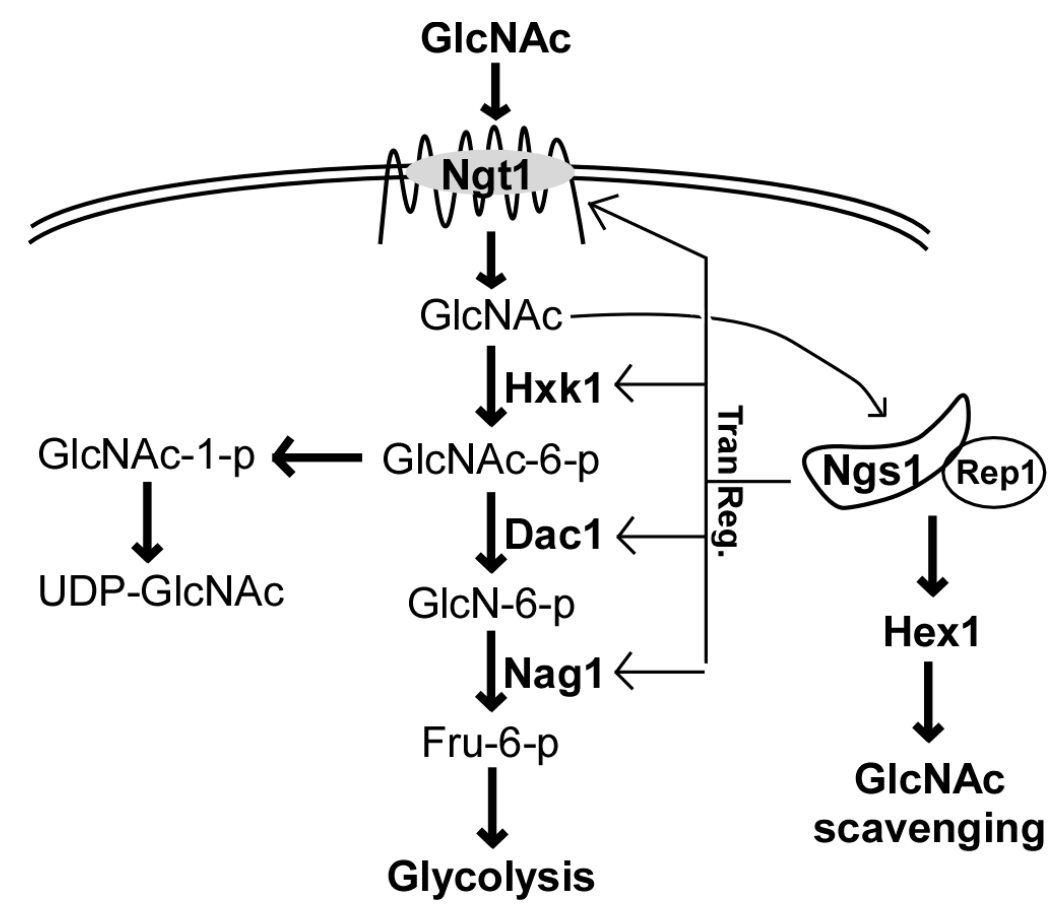

Figure 1. N-acetlyglucosamine (GlcNAc) catabolism in Candida albicans. Ngt1, GlcNAc-specific transporter; Hxk1, GlcNAc kinase; Dac1, GlcNAc-6-phosphate deacetylase; Nag1, Glucosamine-6-phosphate deaminase; Ngs1, N-acetyltransferase; Rep1, transcription factor involved in transcription of GlcNAc-inducible genes. GlcNAc is transported into the cytoplasm by the Ngt1 transporter and is catabolized to fructose-6-phosphate by Hxk1, Dac1, and Nag1. Ngs1 can bind to GlcNAc and may function as a putative sensor. Together with the transcription factor, Rep1, Ngs1 induces the expression of GlcNAc-catabolic genes in the presence of GlcNAc.

\section{GlcNAc Regulates Morphological Transitions and Virulence in Fungi}

Morphological plasticity is a striking characteristic of pathogenic fungi, and many environmental cues, such as $\mathrm{pH}$, temperature, serum, GlcNAc, and $\mathrm{CO}_{2}$, are involved in the regulation of morphological transitions in the Candida clade species [5,29-34]. C. albicans, for example, can transition between several distinct morphological phenotypes [35-39]. Two typical phenotypic switching systems, namely, the yeast-filament transition and white-opaque switching systems, have been extensively studied over the past two decades. Filamentous (hyphal and pseudohyphal) cells are more invasive than yeast-form cells during infections, and the ability to transition between white and opaque cell types is involved in the regulation of both virulence and sexual reproduction in C. albicans [35-39]. Morphological changes are often associated with virulence and adaptation to the continuously changing host environment [40]. The earliest observation for a role of GlcNAc in C. albicans morphological transitions was reported over forty years ago by Simonetti et al. (1974) [4]. The authors found that GlcNAc induced filamentation in C. albicans and hypothesized that GlcNAc is involved in cell wall 
remodeling during morphological transitions, since chitin (containing GlcNAc), is important to cell wall structure and function [4]. Since this initial discovery, significant progress has been made on elucidating the molecular mechanisms involved in GlcNAc-induced filamentation in C. albicans (reviewed by Konopka et al., [1,3]). Filamentation is a key virulence trait in C. albicans that is essential for invasive growth in the host. One intriguing study demonstrated that the administration of GlcNAc in a mouse model of oral candidiasis enhanced host tissue damage and increased the fungal burden of $C$. albicans in the mouse [41]. The authors also observed a dose-dependent effect of GlcNAc concentrations on the severity of the infection in the host. This increase in virulence could be the result of enhanced C. albicans filamentation.

For the white-opaque phenotypic switching system in C. albicans, white and opaque cells differ in colony and cell morphologies, mating competencies, virulence, and transcriptomic profiles [38,40,42,43]. Reversible switching between these two cell types occurs stochastically, yet each cell type is heritable for many generations $[33,44-46]$. The white-opaque switch in C. albicans is controlled by the mating type locus (MTL) [45]. MTL heterozygous cells express the MTLa1/ $\alpha 2$ heterodimer, which binds to the promoter of the opaque-specific master regulator WOR1, repressing its expression and "locking" cells in the white phase [47-50]. Our studies demonstrate that GlcNAc can efficiently induce switching to the opaque cell type in MTL homozygous $(\mathbf{a} / \mathbf{a}$ or $\alpha / \alpha)$ strains, and in a subset of MTL heterozygous $(\mathrm{a} / \alpha)$ clinical isolates [5,51]. Transcription factors Efg1, Rfg1, and Brg1 are involved in the regulation of white-opaque switching in MTLa/ $\alpha$ strains [51-53]. Since opaque cells are mating-competent, switching to the opaque cell type would facilitate mating and genetic recombination in C. albicans. Given that the mammalian gut is a major natural niche for $C$. albicans where commensal bacteria produce GlcNAc and interact with C. albicans, this niche could be a conducive environment for phenotypic switching and possibly mating in C. albicans cells.

C. tropicalis is a member of the Candida clade that is closely related to C. albicans. Interestingly, unlike in C. albicans, GlcNAc represses rather than promotes filamentous growth in C. tropicalis $[9,10]$. However, GlcNAc plays a similar role in the induction of the white-to-opaque transition in C. tropicalis [10]. Overall, these results suggest that GlcNAc plays both distinct and conserved roles in the regulation of morphological transitions in these two and perhaps other Candida species.

Recent studies have shown that GlcNAc also regulates morphology changes and virulence in other fungal species that have pathogenic potential in humans, such as the environmental fungus Cryptococcus neoformans [54] as well as the thermal dimorphic fungi Histoplasma capsulatum and Blastomyces dermatitidis [12]. The production of melanin by C. neoformans is an important virulence factor for this fungal species. Interestingly, GlcNAc affects the cell wall composition, capsule size, and production of melanin in C. neoformans [54]. Although the thermal dimorphic fungi are highly evolutionarily diverged from the Candida species, these studies highlight the conserved function of GlcNAc as a potent inducer of filamentation across diverse fungal species.

\section{GlcNAc Can Induce Morphological Transitions Independent of GlcNAc Catabolism}

The GlcNAc transporter Ngt1 belongs to the major facilitator superfamily (MFS) and is required for the induction of filamentation in C. albicans at low, but not at high, concentrations of GlcNAc [17]. In the closely related species C. tropicalis, GlcNAc suppresses filamentous growth, and consistently, Ngt1 is required for this repression [55]. The thermal dimorphic fugus $H$. capsulatum has two homologs of C. albicans Ngt1, namely Ngt1 and Ngt2, which are required for GlcNAc-induced filamentation [12]. These findings suggest that the mechanisms of GlcNAc-induced morphological transitions are conserved in fungal species with pathogenic potential.

While GlcNAc catabolism is a key virulence trait in C. albicans, it does not appear to be required for GlcNAc-induced filamentation in vitro $[19,56,57]$. Deletion of the GlcNAc catabolizing enzymes HXK1, NAG1, or DAC1 in C. albicans led to failures in the cellular utilization of GlcNAc as a carbon source [56]. GlcNAc also inhibited the growth of nag1/nag1 and dac1/dac1 mutant strains in the presence of other carbon sources, likely due to the toxic effects of accumulated GlcNAc-6-phosphate in the cells [56]. 
Moreover, both the $h x k 1 / h x k 1$ and $h x k 1 / h x k 1$ nag1/nag1 dac1/dac1 triple mutant strains were capable of undergoing filamentation in the presence of GlcNAc [56]. It is also established that the $h x k 1 / h x k 1$ mutant strain is capable of switching to the opaque and gray phenotypes in an MTL-independent manner [58-60]. These results indicate that GlcNAc is involved in the induction of morphological transitions and suggest that GlcNAc-induced morphological transitions are independent of metabolism.

\section{The Ras1-cAMP/PKA Signaling Pathway Functions in GlcNAc-Induced Filamentation}

The conserved Ras1-cAMP/protein kinase A (PKA) signaling pathway is a major regulatory pathway controlling environmental sensing in C. albicans [61-63]. In addition, this pathway also controls morphological transitions, virulence, and stress responses in C. albicans [61-64]. The major components of this pathway include the small GTPase Ras1, the adenylyl cyclase Cyr1, the cyclic nucleotide phosphodiesterases Pde1 and Pde2, the PKA catalytic subunits Tpk1 and Tpk2, and the regulatory subunit Bcy1 [61]. GlcNAc stimulates filamentous growth and white to opaque cell phenotypic switching in C. albicans through activation of the Ras1-cAMP/PKA pathway $[5,57,61,65,66]$. Inactivation of this pathway by either chemical or genetic perturbation suppresses GlcNAc-induced filamentation and white to opaque switching in C. albicans [5,57]. Ras1 is localized to the plasma membranes and/or to the cytoplasm in C. albicans, and constitutive activation of Ras1 via a G13V substitution (Ras1V13) leads to an increase in membrane localization and filamentous growth in the presence of GlcNAc $[65,67]$. The C. albicans adenylyl cyclase Cyr1 contains a Ras-binding domain and multiple domains responsible for sensing distinct environmental signals [64]. Deletion of CYR1 blocks GlcNAc-induced filamentous growth [68], whereas inactivation of the phosphodiesterase PDE2 enhances filamentous growth [69]. Earlier studies showed that the permeable PKA inhibitors, MyrPKI and H-89, inhibit both GlcNAc- and serum-induced filamentation in a concentration-dependent manner [57]. The PKA regulatory subunit Bcy1 acts as a negative regulator of the cAMP signaling pathway $[70,71]$. We previously reported that neither the PKA catalytic subunits (Tpk1 and Tpk2) nor the regulatory subunit (Bcy1) are essential for cell survival in C. albicans [72]. Consistent with the function of Bcy1 in repressing the cAMP/PKA signaling pathway, deletion of $B C Y 1$ results in enhanced filamentation as well as various pleiotropic phenotypes in GlcNAc-containing medium [73].

Other than the Ras1-cAMP/PKA pathway, additional pathways are also likely to regulate GlcNAc-induced filamentation in C. albicans [74,75]. For example, the catabolism of GlcNAc produces ammonia and raises the ambient $\mathrm{pH}$ of the environment. This alkalinization favors the growth of filaments in C. albicans by activating the Rim101-mediated $\mathrm{pH}$ sensing pathway [74,75]. Moreover, this increase in $\mathrm{pH}$ has been shown to counter macrophage acidification during engulfment, thereby allowing C. albicans to survive within macrophages [76].

\section{Ras1-cAMP/PKA Signaling Pathway Functions in GlcNAc-Induced White-Opaque Switching}

The Ras1-cAMP/PKA pathway also regulates white-opaque switching in C. albicans upon environmental changes [33,61]. We found that GlcNAc not only induces white to opaque switching, but that it also stabilizes the opaque cell type at human physiological temperatures (typically, opaque cells would convert en masse to the white cell type under these conditions) [5]. GlcNAc-mediated induction of the opaque cell state occurs primarily through the Ras1-cAMP/PKA pathway [5], while the stability of the opaque state is not dependent on this pathway [77]. Activation of cAMP signaling by constitutive expression of the activated form of Ras1 (Ras1V13), or by mutation of PDE2, led to an en masse conversion to the opaque cell type in the presence of GlcNAc. Consistently, strains with deletions of RAS1 or CYR1 had remarkably decreased white to opaque switch frequencies compared to the isogenic wildtype strain. Wor1, the master regulator of white-opaque switching in C. albicans, is downstream of the Ras1-cAMP/PKA pathway and contains a potential PKA phosphorylation site (T67). Overexpression of WOR1 in Ras1-cAMP/PKA pathway mutant strains overrides their switching defects and promotes the opaque state, however replacing the T67 residue within the PKA consensus motif with alanine (T67A), which cannot be phosphorylated, impairs the function of Wor1 in regulating 
white-opaque switching [5]. Overall, these findings suggest that GlcNAc could regulate white-opaque switching through activation of the Ras1-cAMP/PKA pathway and Wor1 phosphorylation [5].

The transcription factors Wor2 and Flo8 are also important regulators of white-opaque switching in C. albicans, and both are required for inducing switching to the opaque form in medium containing glucose as the sole carbon source $[78,79]$. However, GlcNAc can induce white to opaque switching and stabilize the opaque phenotype in cells of the wor2/wor 2 and flo8/flo 8 mutant strains, indicating that these two regulators can be bypassed via GlcNAc-induced signaling. Interestingly, constitutive overexpression of the activating form of Ras1 (Ras1V13) hypersensitizes cells of the wor2/wor2 mutant strain to GlcNAc [78]. Similar to Wor1, Flo8 is also downstream of the Ras1-cAMP/PKA pathway [80]. It plays critical roles in $\mathrm{CO}_{2}$-induced filamentation and white to opaque switching in C. albicans [79]. $\mathrm{CO}_{2}$ and GlcNAc have synergistic effects on inducing the opaque cell state [5]. Taken together, the Ras1-cAMP/PKA pathway functions as a major regulator of GlcNAc-induced filamentation and white-opaque switching in C. albicans (Figure 2). Finally, there are also other biological processes that are likely regulated by GlcNAc that have yet to be identified.

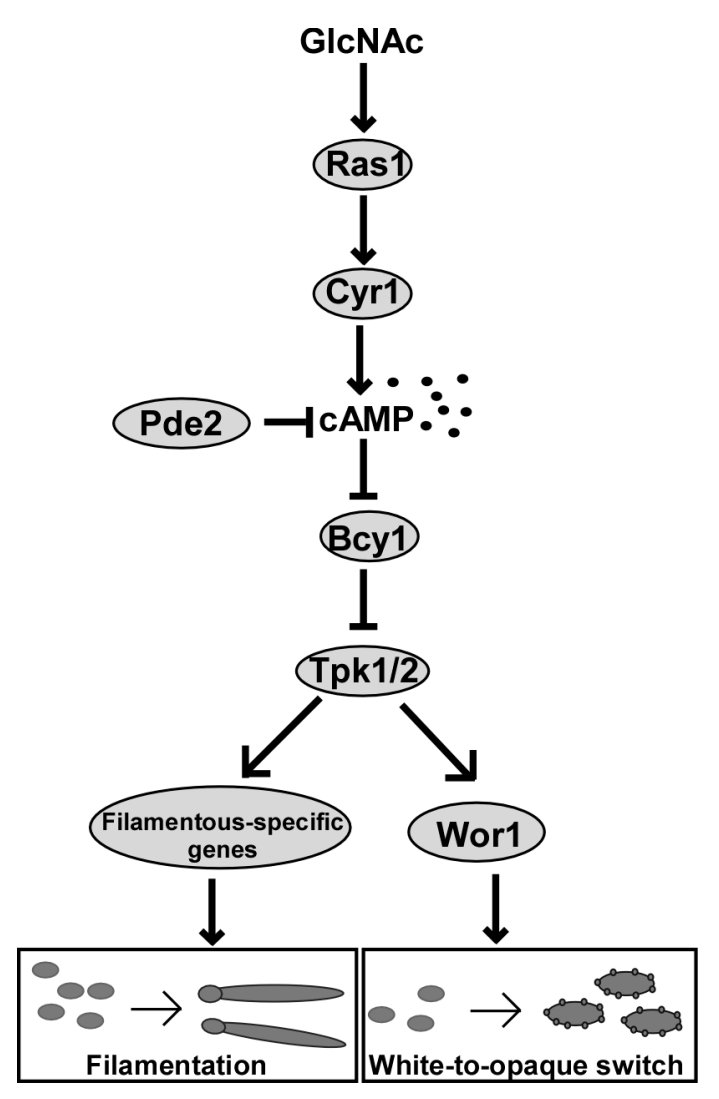

Figure 2. The Ras1-cAMP/PKA signaling pathway regulates GlcNAc-induced filamentation and white-opaque switching in C. albicans. GlcNAc may directly activate the small GTPase Ras1. Activated Ras1 stimulates the adenylyl cyclase Cyr1 to produce cAMP, while the cyclic nucleotide phosphodiesterase Pde2 degrades cAMP. In the absence of GlcNAc, the PKA regulatory subunit Bcy1 binds to the catalytic subunits Tpk1 and Tpk2. In the presence of GlcNAc, cAMP binds to Bcy1 and leads to the release and activation of the catalytic subunits that subsequently phosphorylates filamentation-specific or opaque-specific regulators.

\section{GlcNAc-Induced Cell Death in C. albicans}

For microorganisms, carbon source limitations frequently occur in nature and the ability to sense and respond to nutrient changes is critical for survival. Under nutrient starvation conditions, S. cerevisiae has been shown to enter a G0/G1 phase or to arrest in stationary phase in order to survive [81,82]. The addition of glucose to $S$. cerevisiae cells has been shown to act as a signal for abundant nutrient availability, inducing rapid cell growth [81,82]. However, when incubated in the presence of glucose 
without other nutrients to support growth, S. cerevisiae viability was shown to markedly decrease due to the lack of other nutrients necessary to support growth [81]. Under such conditions, the authors suggest that glucose initiates a new mitotic cell cycle that results in cell death caused by the accumulation of reactive oxygen species (ROS). This glucose-induced cell death in S. cerevisiae is dependent on the cAMP signaling pathway and the rate of sugar phosphorylation $[81,83]$. In general, this phenomenon is called sugar-induced cell death (SICD) and shares characteristics with programmed cell death such as nuclear DNA fragmentation and phosphatidylserine externalization [84].

Interestingly, GlcNAc, but not glucose, induces cell death in C. albicans due to the accumulation of ROS and its associated cell damage [6]. The GI tract, a natural niche for C. albicans, contains many commensal bacteria that contain peptidoglycans in their cell walls $[85,86]$. Although GlcNAc levels within the host have not been directly measured, based on the abundance or peptidoglycan producing bacteria residing in the healthy GI tract, it seems likely that GlcNAc would be an abundant carbon source within this niche. A study by Chang et al. (2004) suggested that GlcNAc is a major nutrient carbon source for Escherichia coli in the mouse gastrointestinal tract [87]. C. albicans may also use GlcNAc rather than glucose, which is scarce in the lower gastrointestinal tract, as a signal for nutrient availability. RNA-Seq analysis demonstrated that GlcNAc treatment increased the expression of ribosomal biogenesis genes in C. albicans, suggesting that protein biosynthesis and metabolism are active, and cells are induced to enter the mitotic phase in the presence of GlcNAc [6]. The cAMP signaling and GlcNAc catabolic pathways are involved in GlcNAc-induced cell death. Although deletion of RAS1 did not affect the efficiency of GlcNAc-induced cell death, inactivation of the adenylyl cyclase-encoding gene CYR1 significantly delayed cell death in C. albicans [6]. Consistent with this finding, activation of the cAMP signaling pathway by constitutive expression of Ras1 (Ras1V13) or by deletion of PDE2 accelerated cell death in the presence of GlcNAc [6]. As expected, deletion of NGT1 or genes of the GlcNAc catabolic pathway also delayed GlcNAc-induced cell death in C. albicans, suggesting that metabolism of GlcNAc is required for this induction. Moreover, it was found that a Candida clade-specific mitochondrial protein, Mcu1, is required for the utilization of GlcNAc in C. albicans. In this study, the deletion of MCU1 resulted in a decrease in susceptibility to GlcNAc and delayed cell death [6]. Furthermore, C. albicans MCU1 was also found to be essential for GlcNAc utilization and filamentation in several filament-inducing conditions as well as for virulence in a mouse systemic infection model [88]. Given the distinct natural niches for C. albicans and S. cerevisiae, it is feasible that these two microorganisms have evolved to detect different carbon sources as signals for nutrient abundance. However, the overall responses to these carbon sources (initiation of cell growth or cell death) are similar between these two distantly related species, suggesting that features of this nutrient response mechanism are conserved.

Interestingly, adjacent to MCU1 is a pseudogene (ORF19.4984) that is predicted to encode a putative LysM domain-containing protein in C. albicans [88]. Fungal LysM domain-containing proteins often bind to chitin or GlcNAc and function as effectors to suppress plant and insect host immune responses [89,90]. The related Candida species, C. tropicalis, is widespread in the environment and is known to contain several genes that encode LysM domain-containing proteins [91,92]. These proteins may aid C. tropicalis in scavenging for and efficiently utilizing environmental chitin as a nutrient source. As a human commensal, C. albicans may not require LysM domain-containing proteins to the same degree as environmental fungi to acquire nutrients in the human host. Taken together, Mcu1 and the putative LysM domain-containing protein may have been functionally associated before ORF19.4984 became a pseudogene and before C. albicans evolved to colonize humans.

\section{Conclusions and Open Questions}

GlcNAc is an abundant carbon source in the environment and within a mammalian host and is utilized as a nutrient and signaling molecule in organisms ranging from bacteria to fungi to humans [1,3]. C. albicans can utilize GlcNAc as a carbon source through its GlcNAc catabolic pathway. In addition to being a nutrient source, GlcNAc functions as a signaling molecule that can induce 
filamentous growth and white to opaque switching in C. albicans. Given that these two morphological transitions have been extensively studied in C. albicans, this fungal species is an ideal model system to further explore the underlying molecular mechanisms of GlcNAc sensing and utilization. Despite the remarkable advances in our understanding of GlcNAc sensing that have been made over the past two decades, many important questions remain to be answered. For example, given the close phylogenetic relationship between C. albicans and C. tropicalis, why does GlcNAc have opposite effects on filamentation in these two fungi? The Ras1-cAMP/PKA signaling pathway is a major regulator of GlcNAc-induced morphological transitions and cell death in C. albicans. Given that Ras1 can be found at both the membrane and the cytoplasm, does GlcNAc directly bind Ras1 and stimulate its activation? Does this binding event affect Ras1 localization? How does GlcNAc signaling, using the same Ras1-cAMP/PKA signaling pathway, regulate different biological processes and phenotypic outputs such as yeast to hyphal growth, white-to-opaque switching, and cell death? Other than its known role as a transporter, does Ngt1 also function as a sensor to detect GlcNAc? Do Ngt1 and Ras1 coordinate to regulate GlcNAc sensing? Does GlcNAc sensing, utilization, and/or the resulting functional outputs of these processes play roles in commensalism? Lastly, how does C. albicans integrate GlcNAc signaling with other environmental signals to respond to changes in the host environment? Mechanistically answering these questions will not only increase our understanding of the survival strategies used by C. albicans in the host, but will also likely lead to the identification of novel antifungal targets for therapeutic development.

Author Contributions: Conceptualization, H.D. and G.H.; writing—original draft preparation, H.D., C.L.E., A.D.H., C.J.N., and G.H.; writing-review and editing, H.D., C.L.E., A.D.H., C.J.N., and G.H.; revising-review and editing, H.D., C.J.N., and G.H. All authors have read and agreed to the published version of the manuscript.

Funding: This research was funded by the National Natural Science Foundation of China awards 31930005 and 31625002 (to G.H.), Natural Science Foundation of Shanghai award 19ZR1405100 (to H.D.), National Institutes of Health (NIH) grants R15AI37975 (to A.D.H.), and R21AI125801 and R35GM124594 (to C.J.N.), and the Kamangar family in the form of an endowed chair (to C.J.N). C.L.E. was supported by NIH fellowship F31DE028488.

Acknowledgments: We thank all members of the Huang, Nobile, and Hernday labs for insightful discussions on the topic of the manuscript.

Conflicts of Interest: The authors declare no conflict of interest. The funders had no role in the design of the study; in the collection, analyses, or interpretation of data; in the writing of the manuscript, or in the decision to publish the results.

\section{References}

1. Konopka, J.B. N-acetylglucosamine (glcnac) functions in cell signaling. Scientifica 2012, $2012,489208$. [CrossRef] [PubMed]

2. Min, K.; Naseem, S.; Konopka, J.B. N-acetylglucosamine regulates morphogenesis and virulence pathways in fungi. J. Fungi 2019, 6, 8. [CrossRef]

3. Naseem, S.; Parrino, S.M.; Buenten, D.M.; Konopka, J.B. Novel roles for GlcNAc in cell signaling. Commun. Integr. Biol. 2012, 5, 156-159. [CrossRef] [PubMed]

4. Simonetti, N.; Strippoli, V.; Cassone, A. Yeast-mycelial conversion induced by N-acetyl-d-glucosamine in Candida albicans. Nature 1974, 250, 344-346. [CrossRef] [PubMed]

5. Huang, G.; Yi, S.; Sahni, N.; Daniels, K.J.; Srikantha, T.; Soll, D.R. N-acetylglucosamine induces white to opaque switching, a mating prerequisite in Candida albicans. PLoS Pathog. 2010, 6, e1000806. [CrossRef]

6. Du, H.; Guan, G.; Li, X.; Gulati, M.; Tao, L.; Cao, C.; Johnson, A.D.; Nobile, C.J.; Huang, G. N-acetylglucosamine -induced cell death in Candida albicans and its implications for adaptive mechanisms of nutrient sensing in yeasts. mBio 2015, 6, e01376-15. [CrossRef]

7. Williams, R.B.; Lorenz, M.C. Multiple alternative carbon pathways combine to promote Candida albicans stress resistance, immune interactions, and virulence. mBio 2020, 11, e03070-19. [CrossRef]

8. Zheng, Q.; Zhang, Q.; Bing, J.; Ding, X.; Huang, G. Environmental and genetic regulation of white-opaque switching in Candida tropicalis. Mol. Microbiol. 2017, 106, 999-1017. [CrossRef] 
9. Zhang, Q.; Tao, L.; Guan, G.; Yue, H.; Liang, W.; Cao, C.; Dai, Y.; Huang, G. Regulation of filamentation in the human fungal pathogen Candida tropicalis. Mol. Microbiol. 2016, 99, 528-545. [CrossRef]

10. Xie, J.; Du, H.; Guan, G.; Tong, Y.; Kourkoumpetis, T.K.; Zhang, L.; Bai, F.Y.; Huang, G. N-acetylglucosamine induces white-to-opaque switching and mating in Candida Tropicalis, providing new insights into adaptation and fungal sexual evolution. Eukaryot. Cell 2012, 11, 773-782. [CrossRef]

11. Perez-Campo, F.M.; Dominguez, A. Factors affecting the morphogenetic switch in Yarrowia lipolytica. Curr. Microbiol. 2001, 43, 429-433. [CrossRef] [PubMed]

12. Gilmore, S.A.; Naseem, S.; Konopka, J.B.; Sil, A. N-acetylglucosamine (glcnac) triggers a rapid, temperatureresponsive morphogenetic program in thermally dimorphic fungi. PLoS Genet. 2013, 9, e1003799. [CrossRef] [PubMed]

13. Pfaller, M.A.; Diekema, D.J.; Turnidge, J.D.; Castanheira, M.; Jones, R.N. Twenty years of the sentry antifungal surveillance program: Results for Candida species from 1997-2016. Open Forum Infect. Dis. 2019, 6, S79-S94. [CrossRef] [PubMed]

14. Pfaller, M.A.; Andes, D.R.; Diekema, D.J.; Horn, D.L.; Reboli, A.C.; Rotstein, C.; Franks, B.; Azie, N.E. Epidemiology and outcomes of invasive candidiasis due to non-albicans species of Candida in 2,496 patients: Data from the prospective antifungal therapy (path) registry 2004-2008. PLoS ONE 2014, 9, e101510. [CrossRef]

15. Odds, F.C. Molecular phylogenetics and epidemiology of Candida albicans. Future Microbiol. 2010, 5, 67-79. [CrossRef]

16. Berman, J. Candida albicans. Curr. Biol. 2012, 22, R620-R622. [CrossRef]

17. Alvarez, F.J.; Konopka, J.B. Identification of an N-acetylglucosamine transporter that mediates hyphal induction in Candida albicans. Mol. Biol. Cell. 2007, 18, 965-975. [CrossRef]

18. Lorenz, M.C.; Bender, J.A.; Fink, G.R. Transcriptional response of Candida albicans upon internalization by macrophages. Eukaryot. Cell 2004, 3, 1076-1087. [CrossRef]

19. Kumar, M.J.; Jamaluddin, M.S.; Natarajan, K.; Kaur, D.; Datta, A. The inducible N-acetylglucosamine catabolic pathway gene cluster in Candida albicans: Discrete N-acetylglucosamine-inducible factors interact at the promoter of nag1. Proc. Natl. Acad. Sci. USA 2000, 97, 14218-14223. [CrossRef]

20. Nadal, M.; Sawers, R.; Naseem, S.; Bassin, B.; Kulicke, C.; Sharman, A.; An, G.; An, K.; Ahern, K.R.; Romag, A.; et al. An $\mathrm{N}$-acetylglucosamine transporter required for arbuscular mycorrhizal symbioses in rice and maize. Nat. Plants 2017, 3, 17073. [CrossRef]

21. Kappel, L.; Gaderer, R.; Flipphi, M.; Seidl-Seiboth, V. The N-acetylglucosamine catabolic gene cluster in Trichoderma reesei is controlled by the ndt80-like transcription factor ron1. Mol. Microbiol. 2016, 99, 640-657. [CrossRef] [PubMed]

22. Yamada-Okabe, T.; Sakamori, Y.; Mio, T.; Yamada-Okabe, H. Identification and characterization of the genes for $\mathrm{n}$-acetylglucosamine kinase and $\mathrm{N}$-acetylglucosamine-phosphate deacetylase in the pathogenic fungus Candida albicans. Eur. J. Biochem. 2001, 268, 2498-2505. [CrossRef] [PubMed]

23. Natarajan, K.; Datta, A. Molecular cloning and analysis of the nag1 cdna coding for glucosamine-6-phosphate deaminase from Candida albicans. J. Biol. Chem. 1993, 268, 9206-9214. [PubMed]

24. Naseem, S.; Min, K.; Spitzer, D.; Gardin, J.; Konopka, J.B. Regulation of hyphal growth and N-acetylglucosamine catabolism by two transcription factors in Candida albicans. Genetics 2017, 206, 299-314. [CrossRef] [PubMed]

25. Su, C.; Lu, Y.; Liu, H. N-acetylglucosamine sensing by a gen5-related n-acetyltransferase induces transcription via chromatin histone acetylation in fungi. Nat. Commun. 2016, 7, 12916. [CrossRef] [PubMed]

26. Ruhela, D.; Kamthan, M.; Saha, P.; Majumdar, S.S.; Datta, K.; Abdin, M.Z.; Datta, A. In vivo role of Candida albicans beta-hexosaminidase (Hex1) in carbon scavenging. MicrobiologyOpen 2015, 4, 730-742. [CrossRef]

27. Cannon, R.D.; Niimi, K.; Jenkinson, H.F.; Shepherd, M.G. Molecular cloning and expression of the Candida albicans beta-N-acetylglucosaminidase (hex1) gene. J. Bacteriol. 1994, 176, 2640-2647. [CrossRef]

28. Min, K.; Biermann, A.; Hogan, D.A.; Konopka, J.B. Genetic analysis of Ndt80 family transcription factors in Candida albicans using new crispr-CAS9 approaches. mSphere 2018, 3, e00545-18. [CrossRef]

29. Klengel, T.; Liang, W.J.; Chaloupka, J.; Ruoff, C.; Schroppel, K.; Naglik, J.R.; Eckert, S.E.; Mogensen, E.G.; Haynes, K.; Tuite, M.F.; et al. Fungal adenylyl cyclase integrates $\mathrm{CO}_{2}$ sensing with cAMP signaling and virulence. Curr. Biol. 2005, 15, 2021-2026. [CrossRef]

30. Huang, G.; Srikantha, T.; Sahni, N.; Yi, S.; Soll, D.R. CO $\mathrm{CO}_{2}$ regulates white-to-opaque switching in Candida albicans. Curr. Biol. 2009, 19, 330-334. [CrossRef] 
31. Davis, D. Adaptation to environmental ph in Candida albicans and its relation to pathogenesis. Curr. Genet. 2003, 44, 1-7. [CrossRef] [PubMed]

32. Davis, D.; Wilson, R.B.; Mitchell, A.P. Rim101-dependent and-independent pathways govern ph responses in Candida albicans. Mol. Cell. Biol. 2000, 20, 971-978. [CrossRef] [PubMed]

33. Huang, G. Regulation of phenotypic transitions in the fungal pathogen Candida albicans. Virulence 2012, 3, 251-261. [CrossRef] [PubMed]

34. Biswas, S.; Van Dijck, P.; Datta, A. Environmental sensing and signal transduction pathways regulating morphopathogenic determinants of Candida albicans. Microbiol. Mol. Biol. Rev. 2007, 71, 348-376. [CrossRef]

35. Tao, L.; Du, H.; Guan, G.; Dai, Y.; Nobile, C.J.; Liang, W.; Cao, C.; Zhang, Q.; Zhong, J.; Huang, G. Discovery of a "white-gray-opaque" tristable phenotypic switching system in Candida albicans: Roles of non-genetic diversity in host adaptation. PLoS Biol. 2014, 12, e1001830. [CrossRef] [PubMed]

36. Pande, K.; Chen, C.; Noble, S.M. Passage through the mammalian gut triggers a phenotypic switch that promotes Candida albicans commensalism. Nat. Genet. 2013, 45, 1088-1091. [CrossRef] [PubMed]

37. Jain, N.; Hasan, F.; Fries, B.C. Phenotypic switching in fungi. Curr. Fungal Inf. Rep. 2008, 2, 180-188. [CrossRef]

38. Slutsky, B.; Staebell, M.; Anderson, J.; Risen, L.; Pfaller, M.; Soll, D.R. White-opaque transition: A second high-frequency switching system in Candida albicans. J. Bacteriol. 1987, 169, 189-197. [CrossRef]

39. Slutsky, B.; Buffo, J.; Soll, D.R. High-frequency switching of colony morphology in Candida albicans. Science 1985, 230, 666-669. [CrossRef]

40. Noble, S.M.; Gianetti, B.A.; Witchley, J.N. Candida albicans cell-type switching and functional plasticity in the mammalian host. Nat. Rev. Microbiol. 2017, 15, 96-108. [CrossRef]

41. Ishijima, S.A.; Hayama, K.; Takahashi, M.; Holmes, A.R.; Cannon, R.D.; Abe, S. N-acetylglucosamine increases symptoms and fungal burden in a murine model of oral candidiasis. Med. Mycol. 2012, 50, 252-258. [CrossRef] [PubMed]

42. Lan, C.Y.; Newport, G.; Murillo, L.A.; Jones, T.; Scherer, S.; Davis, R.W.; Agabian, N. Metabolic specialization associated with phenotypic switching in candidaalbicans. Proc. Natl. Acad. Sci. USA 2002, 99, 14907-14912. [CrossRef]

43. Soll, D.R. Why does Candida albicans switch? FEMS Yeast Res. 2009, 9, 973-989. [CrossRef] [PubMed]

44. Liang, W.; Guan, G.; Li, C.; Nobile, C.J.; Tao, L.; Huang, G. Genetic regulation of the development of mating projections in Candida albicans. Emerg. Microbes Infect. 2020, 9, 413-426. [CrossRef] [PubMed]

45. Miller, M.G.; Johnson, A.D. White-opaque switching in Candida albicans is controlled by mating-type locus homeodomain proteins and allows efficient mating. Cell 2002, 110, 293-302. [CrossRef]

46. Anderson, J.M.; Soll, D.R. Unique phenotype of opaque cells in the white-opaque transition of Candida albicans. J. Bacteriol. 1987, 169, 5579-5588. [CrossRef]

47. Zordan, R.E.; Miller, M.G.; Galgoczy, D.J.; Tuch, B.B.; Johnson, A.D. Interlocking transcriptional feedback loops control white-opaque switching in Candida albicans. PLoS Biol. 2007, 5, e256. [CrossRef]

48. Zordan, R.E.; Galgoczy, D.J.; Johnson, A.D. Epigenetic properties of white-opaque switching in Candida albicans are based on a self-sustaining transcriptional feedback loop. Proc. Natl. Acad. Sci. USA 2006, 103, 12807-12812. [CrossRef]

49. Srikantha, T.; Borneman, A.R.; Daniels, K.J.; Pujol, C.; Wu, W.; Seringhaus, M.R.; Gerstein, M.; Yi, S.; Snyder, M.; Soll, D.R. Tos9 regulates white-opaque switching in Candida albicans. Eukaryot. Cell 2006, 5, 1674-1687. [CrossRef]

50. Huang, G.; Wang, H.; Chou, S.; Nie, X.; Chen, J.; Liu, H. Bistable expression of Wor1, a master regulator of white-opaque switching in Candida albicans. Proc. Natl. Acad. Sci. USA 2006, 103, 12813-12818. [CrossRef]

51. Xie, J.; Tao, L.; Nobile, C.J.; Tong, Y.; Guan, G.; Sun, Y.; Cao, C.; Hernday, A.D.; Johnson, A.D.; Zhang, L.; et al. White-opaque switching in natural MTLa/alpha isolates of Candida albicans: Evolutionary implications for roles in host adaptation, pathogenesis, and sex. PLoS Biol. 2013, 11, e1001525. [CrossRef] [PubMed]

52. Park, Y.N.; Conway, K.; Pujol, C.; Daniels, K.J.; Soll, D.R. Efg1 mutations, phenotypic switching, and colonization by clinical a/alpha strains of Candida albicans. mSphere 2020, 5, e00795-19. [CrossRef] [PubMed]

53. Park, Y.N.; Conway, K.; Conway, T.P.; Daniels, K.J.; Soll, D.R. Roles of the transcription factors Sfl2 and Efg1 in white-opaque switching in a/alpha strains of Candida albicans. mSphere 2019, 4, e00703-18. [CrossRef] [PubMed] 
54. Camacho, E.; Chrissian, C.; Cordero, R.J.B.; Liporagi-Lopes, L.; Stark, R.E.; Casadevall, A. N-acetylglucosamine affects Cryptococcus Neoformans cell-wall composition and melanin architecture. Microbiology 2017, 163, 1540-1556. [CrossRef] [PubMed]

55. Zhang, Q.; Xu, L.; Yuan, S.; Zhou, Q.; Wang, X.; Wang, L.; Hu, Z.; Yan, Y. Ngt1 is essential for n-acetylglucosamine-mediated filamentous growth inhibition and Hxk1 functions as a positive regulator of filamentous growth in candida tropicalis. Int. J. Mol. Sci. 2020, 21, 4036. [CrossRef]

56. Naseem, S.; Gunasekera, A.; Araya, E.; Konopka, J.B. N-acetylglucosamine (GlcNAc) induction of hyphal morphogenesis and transcriptional responses in Candida albicans are not dependent on its metabolism. J. Biol. Chem. 2011, 286, 28671-28680. [CrossRef]

57. Castilla, R.; Passeron, S.; Cantore, M.L. N-acetyl-d-glucosamine induces germination in Candida albicans through a mechanism sensitive to inhibitors of cAMP-dependent protein kinase. Cell. Signal. 1998, 10, 713-719. [CrossRef]

58. Cao, C.; Guan, G.; Du, H.; Tao, L.; Huang, G. Role of the n-acetylglucosamine kinase (hxk1) in the regulation of white-gray-opaque tristable phenotypic transitions in c. Albicans. Fungal Genet. Biol. FG B 2016, 92, $26-32$. [CrossRef]

59. Rao, K.H.; Ruhela, D.; Ghosh, S.; Abdin, M.Z.; Datta, A. N-acetylglucosamine kinase, Hxk1 contributes to white-opaque morphological transition in Candida albicans. Biochem. Biophys. Res. Commun. 2014, 445, 138-144. [CrossRef]

60. Rao, K.H.; Ghosh, S.; Natarajan, K.; Datta, A. N-acetylglucosamine kinase, Hxk1 is involved in morphogenetic transition and metabolic gene expression in Candida albicans. PLoS ONE 2013, 8, e53638. [CrossRef]

61. Huang, G.; Huang, Q.; Wei, Y.; Wang, Y.; Du, H. Multiple roles and diverse regulation of the r Ras/cAMP/protein kinase A pathway in Candida albicans. Mol. Microbiol. 2019, 111, 6-16. [CrossRef] [PubMed]

62. Hogan, D.A.; Sundstrom, P. The Ras/cAMP/PKA signaling pathway and virulence in Candida albicans. Future Microbiol. 2009, 4, 1263-1270. [CrossRef] [PubMed]

63. Fimia, G.M.; Sassone-Corsi, P. Cyclic AMP signalling. J. Cell Sci. 2001, 114, 1971-1972.

64. Wang, Y. Fungal adenylyl cyclase acts as a signal sensor and integrator and plays a central role in interaction with bacteria. PLoS Pathog. 2013, 9, e1003612. [CrossRef]

65. Piispanen, A.E.; Bonnefoi, O.; Carden, S.; Deveau, A.; Bassilana, M.; Hogan, D.A. Roles of ras1 membrane localization during Candida albicans hyphal growth and farnesol response. Eukaryot. Cell 2011, 10, 1473-1484. [CrossRef] [PubMed]

66. Cloutier, M.; Castilla, R.; Bolduc, N.; Zelada, A.; Martineau, P.; Bouillon, M.; Magee, B.B.; Passeron, S.; Giasson, L.; Cantore, M.L. The two isoforms of the cAMP-dependent protein kinase catalytic subunit are involved in the control of dimorphism in the human fungal pathogen Candida albicans. Fungal Gen. Biol. FG B 2003, 38, 133-141. [CrossRef]

67. Inglis, D.O.; Sherlock, G. Ras signaling gets fine-tuned: Regulation of multiple pathogenic traits of Candida albicans. Eukaryot. Cell 2013, 12, 1316-1325. [CrossRef]

68. Rocha, C.R.; Schroppel, K.; Harcus, D.; Marcil, A.; Dignard, D.; Taylor, B.N.; Thomas, D.Y.; Whiteway, M.; Leberer, E. Signaling through adenylyl cyclase is essential for hyphal growth and virulence in the pathogenic fungus Candida albicans. Mol. Biol. Cell 2001, 12, 3631-3643. [CrossRef]

69. Jung, W.H.; Warn, P.; Ragni, E.; Popolo, L.; Nunn, C.D.; Turner, M.P.; Stateva, L. Deletion of PDE2, the gene encoding the high-affinity camp phosphodiesterase, results in changes of the cell wall and membrane in Candida albicans. Yeast 2005, 22, 285-294. [CrossRef]

70. Giacometti, R.; Souto, G.; Silberstein, S.; Giasson, L.; Cantore, M.L.; Passeron, S. Expression levels and subcellular localization of bcy1p in Candida albicans mutant strains devoid of one $B C Y 1$ allele results in a defective morphogenetic behavior. Biochim. Biophys. Acta 2006, 1763, 64-72. [CrossRef]

71. Toda, T.; Cameron, S.; Sass, P.; Zoller, M.; Scott, J.D.; McMullen, B.; Hurwitz, M.; Krebs, E.G.; Wigler, M. Cloning and characterization of bcy1, a locus encoding a regulatory subunit of the cyclic AMP-dependent protein kinase in Saccharomyces cerevisiae. Mol. Cell Biol. 1987, 7, 1371-1377. [CrossRef] [PubMed]

72. Bockmuhl, D.P.; Krishnamurthy, S.; Gerads, M.; Sonneborn, A.; Ernst, J.F. Distinct and redundant roles of the two protein kinase a isoforms Tpk1p and Tpk2p in morphogenesis and growth of Candida albicans. Mol. Microbiol. 2001, 42, 1243-1257. [CrossRef] [PubMed] 
73. Ding, X.; Cao, C.; Zheng, Q.; Huang, G. The regulatory subunit of protein kinase a (bcy1) in Candida albicans plays critical roles in filamentation and white-opaque switching but is not essential for cell growth. Front. Microbiol. 2016, 7, 2127. [CrossRef] [PubMed]

74. Parrino, S.M.; Si, H.; Naseem, S.; Groudan, K.; Gardin, J.; Konopka, J.B. cAMP-independent signal pathways stimulate hyphal morphogenesis in Candida albicans. Mol. Microbiol. 2017, 103, 764-779. [CrossRef]

75. Naseem, S.; Araya, E.; Konopka, J.B. Hyphal growth in Candida albicans does not require induction of hyphal-specific gene expression. Mol. Biol. Cell 2015, 26, 1174-1187. [CrossRef]

76. Vesely, E.M.; Williams, R.B.; Konopka, J.B.; Lorenz, M.C. N-acetylglucosamine metabolism promotes survival of Candida albicans in the phagosome. $m$ Sphere 2017, 2, e00357-17. [CrossRef]

77. Cao, C.; Wu, M.; Bing, J.; Tao, L.; Ding, X.; Liu, X.; Huang, G. Global regulatory roles of the cAMP/PKA pathway revealed by phenotypic, transcriptomic and phosphoproteomic analyses in a null mutant of the PKA catalytic subunit in Candida albicans. Mol. Microbiol. 2017, 105, 46-64. [CrossRef]

78. Tong, Y.; Cao, C.; Xie, J.; Ni, J.; Guan, G.; Tao, L.; Zhang, L.; Huang, G. N-acetylglucosamine-induced white-to-opaque switching in Candida albicans is independent of the Wor2 transcription factor. Fungal Gen. Biol. FG B 2014, 62, 71-77. [CrossRef]

79. Du, H.; Guan, G.; Xie, J.; Cottier, F.; Sun, Y.; Jia, W.; Muhlschlegel, F.A.; Huang, G. The transcription factor Flo8 mediates $\mathrm{CO}_{2}$ sensing in the human fungal pathogen Candida albicans. Mol. Biol. Cell 2012, 23, $2692-2701$. [CrossRef]

80. Cao, F.; Lane, S.; Raniga, P.P.; Lu, Y.; Zhou, Z.; Ramon, K.; Chen, J.; Liu, H. The flo8 transcription factor is essential for hyphal development and virulence in Candida albicans. Mol. Biol. Cell 2006, 17, $295-307$. [CrossRef]

81. Granot, D.; Snyder, M. Glucose induces cAMP-independent growth-related changes in stationary-phase cells of Saccharomyces cerevisiae. Proc. Natl. Acad. Sci. USA 1991, 88, 5724-5728. [CrossRef] [PubMed]

82. Unger, M.W.; Hartwell, L.H. Control of cell division in Saccharomyces Cerevisiae by methionyl-trna. Proc. Natl. Acad. Sci. USA 1976, 73, 1664-1668. [CrossRef] [PubMed]

83. Granot, D.; Dai, N. Sugar induced cell death in yeast is dependent on the rate of sugar phosphorylation as determined by Arabidopsis Thaliana hexokinase. Cell Death Differ. 1997, 4, 555-559. [CrossRef] [PubMed]

84. Granot, D.; Levine, A.; Dor-Hefetz, E. Sugar-induced apoptosis in yeast cells. FEMS Yeast Res. 2003, 4, 7-13. [CrossRef]

85. Clarke, T.B.; Davis, K.M.; Lysenko, E.S.; Zhou, A.Y.; Yu, Y.; Weiser, J.N. Recognition of peptidoglycan from the microbiota by Nod1 enhances systemic innate immunity. Nat. Med. 2010, 16, 228-231. [CrossRef]

86. Belkaid, Y.; Hand, T.W. Role of the microbiota in immunity and inflammation. Cell 2014, 157, 121-141. [CrossRef]

87. Chang, D.E.; Smalley, D.J.; Tucker, D.L.; Leatham, M.P.; Norris, W.E.; Stevenson, S.J.; Anderson, A.B.; Grissom, J.E.; Laux, D.C.; Cohen, P.S.; et al. Carbon nutrition of Escherichia coli in the mouse intestine. Proc. Natl. Acad. Sci. USA 2004, 101, 7427-7432. [CrossRef]

88. Guan, G.; Wang, H.; Liang, W.; Cao, C.; Tao, L.; Naseem, S.; Konopka, J.B.; Wang, Y.; Huang, G. The mitochondrial protein mcu1 plays important roles in carbon source utilization, filamentation, and virulence in Candida albicans. Fungal Gen. Biol. FG B 2015, 81, 150-159. [CrossRef]

89. de Jonge, R.; Thomma, B.P. Fungal lysm effectors: Extinguishers of host immunity? Trends Microbiol. 2009, 17, 151-157. [CrossRef]

90. Buist, G.; Steen, A.; Kok, J.; Kuipers, O.P. Lysm, a widely distributed protein motif for binding to (peptido) glycans. Mol. Microbiol. 2008, 68, 838-847. [CrossRef]

91. Kutty, S.N.; Philip, R. Marine yeasts-a review. Yeast 2008, 25, 465-483. [CrossRef] [PubMed]

92. Hagler, A.N.; Mendonca-Hagler, L.C. Yeasts from marine and estuarine waters with different levels of pollution in the state of rio de janeiro, brazil. Appl. Environ. Microbiol. 1981, 41, 173-178. [CrossRef] [PubMed]

(C) 2020 by the authors. Licensee MDPI, Basel, Switzerland. This article is an open access article distributed under the terms and conditions of the Creative Commons Attribution (CC BY) license (http://creativecommons.org/licenses/by/4.0/). 